\title{
On the stability of the linear functional equation in a single variable on complete metric groups
}

\author{
Soon-Mo Jung, Dorian Popa, Michael Th. Rassias \\ Mathematics Section, College of Science and Technology, \\ Hongik University, 339-701 Sejong, Republic of Korea \\ E-mail: smjung@hongik.ac.kr \\ Department of Mathematics, Technical University of Cluj-Napoca, \\ 28 Memorandumului, 400114 Cluj-Napoca, Romania \\ E-mail: Popa.Dorian@math.utcluj.ro \\ Department of Mathematics, ETH-Zürich, Ramistrasse 101, \\ 8092 Zürich, Switzerland \\ E-mail: michail.rassias@math.ethz.ch
}

\begin{abstract}
In this paper we obtain a result on Hyers-Ulam stability of the linear functional equation in a single variable $f(\varphi(x))=g(x) \cdot f(x)$ on a complete metric group.
\end{abstract}

\section{Introduction}

Hyers-Ulam stability is one of the main topics in the theory of functional equations. Generally a functional equation is said to be stable provided, for any function $f$ satisfying the perturbed functional equation, there exists an exact solution $f_{0}$ of that equation which is not far from the

\footnotetext{
${ }^{0}$ Mathematics Subject Classification (2010): 65J15.

${ }^{0}$ Key words and phrases: Nonlinear operator, stability, functional equation, complete metric group, inequalities, Banach spaces, operator mapping, Euler-Mascheroni constant.
} 
given $f$. Based on this concept, the study of the stability of functional equations can be regarded as a branch of optimization theory. (We can find some applications of the Hyers-Ulam stability to optimization theory and economics in [11.)

It seems that the first result on the stability of functional equations appeared in the famous book by Gy. Pólya and G. Szegö [18] and concerns the Cauchy functional equation on the set of positive integers. But the starting point of the stability theory of functional equations is due to S.M. Ulam who formulated a question concerning the perturbation of homomorphisms on metric groups. The first result for Ulam's problem was obtained by D.H. Hyers for the Cauchy functional equation on Banach spaces. Due to the question of Ulam and the answer of Hyers the stability of functional equations is called after their names.

For more details on Hyers-Ulam stability of functional equations and optimization theory we refer the reader to [2, 4, 12, 13, 15, 17, 19].

The functional equation

$$
f(\varphi(x))=g(x) f(x)+h(x)
$$

where $f$ is the unknown function and $g, h, \varphi$ are given functions, is called the linear functional equation in a single variable. For particular cases of $g$ and $h$ in (1.1) we obtain some classical functional equations. We mention here some of them as

- Abel's equation

$$
f(\varphi(x))=f(x)+c
$$

- Schröder's equation

$$
f(\varphi(x))=c f(x)
$$

- Gamma functional equation

$$
f(x+1)=x f(x)
$$

- Digamma functional equation

$$
f(x+1)=f(x)+\frac{1}{x} .
$$


Recall that Digamma function $\psi_{0}: \mathbb{R}_{+}^{*} \rightarrow \mathbb{R}$ is defined by

$$
\psi_{0}(x)=\frac{d}{d x} \ln \Gamma(x)=\frac{\Gamma^{\prime}(x)}{\Gamma(x)}, \quad \forall x \in \mathbb{R}_{+}^{*},
$$

where

$$
\Gamma(x)=\int_{0}^{\infty} t^{x-1} e^{-t} d t, \quad \forall x \in \mathbb{R}_{+}^{*},
$$

$\mathbb{R}_{+}$stands for the set of all nonnegative numbers, i.e., $\mathbb{R}_{+}=[0, \infty)$ and $\mathbb{R}_{+}^{*}=(0, \infty)$. For more details on the functional equation (1.1) and its particular cases we refer to [16] and the references therein. It seems that the first result on stability for the equation (1.1) was obtained in 1970 by J. Brydak [3]. A generalized Hyers-Ulam stability of the gamma functional equation was obtained by S.-M. Jung in [14]. A nice result on generalized Hyers-Ulam stability of the equation (1.1) was obtained by T. Trif [20] for functions $f$ acting from an arbitrary nonempty set $S$ into a Banach space $X$.

Some recent results on the stability and nonstability of the equation (1.1) and the linear functional equation of higher order in a single variable were obtained by J. Brzdek, D. Popa, B. Xu (see [6, 7, 8, 9, 10]).

The goal of this paper is to study the Hyers-Ulam stability of the homogeneous linear functional equation (1.1) for functions defined from an arbitrary nonempty set $S$ into a complete metric group $(G, \cdot, d)$, i.e., $(G, \cdot)$ is a group, $(G, d)$ is a complete metric space, the group's binary operation and the inverse operation are continuous with respect to the product topology on $G \times G$ and the topology generated by the metric $d$ on $G$, respectively.

\section{Stability of linear functional equation}

Let $S$ be a nonempty set, $(G, \cdot, d)$ a complete metric group with the metric $d$ invariant to left translations, i.e.,

$$
d(x \cdot y, x \cdot z)=d(y, z), \quad \forall x, y, z \in G,
$$

and let $\varphi: S \rightarrow S, g: S \rightarrow G$ be given functions. An example of metric invariant to left translations is the metric induced by a norm. 
We deal with the Hyers-Ulam stability of the linear functional equation

$$
f(\varphi(x))=g(x) \cdot f(x),
$$

where $f: S \rightarrow G$ is the unknown function.

Let $\mathbb{R}_{+}^{S}$ be the class of all functions $\varepsilon: S \rightarrow \mathbb{R}_{+}$. We study the generalized Hyers-Ulam stability of the equation (2.2) in the sense defined in [4].

Definition 2.1 Let $\mathcal{C} \subseteq \mathbb{R}_{+}^{S}$ be nonempty and $\mathcal{T}$ be an operator mapping $\mathcal{C}$ into $\mathbb{R}_{+}^{S}$. We say that the equation $(2.2)$ is $\mathcal{T}$-stable (with uniqueness, respectively) provided for every $\varepsilon \in \mathcal{C}$ and $f: S \rightarrow G$ with

$$
d(f(\varphi(x)), g(x) \cdot f(x)) \leq \varepsilon(x), \quad \forall x \in S
$$

there exists a (unique, respectively) solution $f_{0}: S \rightarrow G$ of the equation (2.2) such that

$$
d\left(f(x), f_{0}(x)\right) \leq \mathcal{T} \varepsilon(x), \quad \forall x \in S
$$

If $\varepsilon$ is a constant function in the previous definition then the equation (2.2) is said to be stable in Hyers-Ulam sense.

By $\varphi^{k}, k \in \mathbb{N}_{0}=\mathbb{N} \cup\{0\}$ we denote the $k$-th iterate of the function $\varphi, \varphi^{0}=1_{S}, \varphi^{k}=\varphi \circ \varphi^{k-1}, k \in \mathbb{N}$.

The main result is contained in the next theorem.

Theorem 2.2 Let $\varepsilon: S \rightarrow \mathbb{R}_{+}$be a function with the property

$$
\sum_{n=0}^{\infty} \varepsilon\left(\varphi^{n}(x)\right)=\Phi(x), \quad \forall x \in S,
$$

where $\Phi: S \rightarrow \mathbb{R}_{+}$. Then for every function $f: S \rightarrow G$ satisfying the inequality

$$
d(f(\varphi(x)), g(x) \cdot f(x)) \leq \varepsilon(x), \quad \forall x \in S,
$$

there exists a unique solution $f_{0}: S \rightarrow G$ of the functional equation (2.2) such that

$$
d\left(f(x), f_{0}(x)\right) \leq \Phi(x), \quad \forall x \in S .
$$


Proof. Existence. Let $f: S \rightarrow G$ be a function satisfying (2.4). Then the following relation holds:

$$
d\left(f\left(\varphi^{n}(x)\right), \prod_{k=1}^{n} g\left(\varphi^{k-1}(x)\right) \cdot f(x)\right) \leq \sum_{k=1}^{n} \varepsilon\left(\varphi^{k-1}(x)\right)
$$

for all $x \in S$ and $n \in \mathbb{N}$. We prove (2.6) by induction on $n$. Since the group $(G, \cdot)$ is not generally commutative, we let

$$
\prod_{k=p}^{n} a_{k}:=a_{n} \cdot a_{n-1} \cdot \ldots \cdot a_{p}
$$

where $a_{k} \in G$ for $p \leq k \leq n$.

For $n=1$ the relation (2.6) holds in view of (2.4). We suppose that (2.6) holds for some $n \in \mathbb{N}$ and for all $x \in S$, and we prove that

$$
d\left(f\left(\varphi^{n+1}(x)\right), \prod_{k=1}^{n+1} g\left(\varphi^{k-1}(x)\right) \cdot f(x)\right) \leq \sum_{k=1}^{n+1} \varepsilon\left(\varphi^{k-1}(x)\right), \quad x \in S .
$$

Indeed, it follows from (2.1), (2.4) and (2.6) that

$$
\begin{aligned}
& d\left(f\left(\varphi^{n+1}(x)\right), \prod_{k=1}^{n+1} g\left(\varphi^{k-1}(x)\right) \cdot f(x)\right) \\
& \leq d\left(f\left(\varphi^{n+1}(x)\right), g\left(\varphi^{n}(x)\right) \cdot f\left(\varphi^{n}(x)\right)\right) \\
& \quad+d\left(g\left(\varphi^{n}(x)\right) \cdot f\left(\varphi^{n}(x)\right), \prod_{k=1}^{n+1} g\left(\varphi^{k-1}(x)\right) \cdot f(x)\right) \\
& \leq \varepsilon\left(\varphi^{n}(x)\right)+d\left(f\left(\varphi^{n}(x)\right), \prod_{k=1}^{n} g\left(\varphi^{k-1}(x)\right) \cdot f(x)\right) \\
& \leq \sum_{k=1}^{n+1} \varepsilon\left(\varphi^{k-1}(x)\right), \quad x \in S .
\end{aligned}
$$

Hence (2.6) holds for all $x \in S$ and $n \in \mathbb{N}$.

Now let $\left(\varepsilon_{n}\right)_{n \geq 1}$ be the sequence of functions defined by

$$
\varepsilon_{n}(x):=\left(\prod_{k=1}^{n} g\left(\varphi^{k-1}(x)\right)\right)^{-1} \cdot f\left(\varphi^{n}(x)\right), \quad n \in \mathbb{N}, x \in S .
$$


We prove that $\left(\varepsilon_{n}(x)\right)_{n \geq 1}$ is a Cauchy sequence in $(G, \cdot d)$ for all $x \in S$, where $a^{-1}$ means the inverse of the element $a$ in the group $G$. Using (2.1) and (2.6), we have

$$
\begin{aligned}
& d\left(\varepsilon_{n+p}(x), \varepsilon_{n}(x)\right) \\
& =d\left(\left(\prod_{k=1}^{n+p} g\left(\varphi^{k-1}(x)\right)\right)^{-1} \cdot f\left(\varphi^{n+p}(x)\right),\left(\prod_{k=1}^{n} g\left(\varphi^{k-1}(x)\right)\right)^{-1} \cdot f\left(\varphi^{n}(x)\right)\right) \\
& =d\left(\left(\prod_{k=n+1}^{n+p} g\left(\varphi^{k-1}(x)\right)\right)^{-1} \cdot f\left(\varphi^{n+p}(x)\right), f\left(\varphi^{n}(x)\right)\right) \\
& \leq \sum_{k=1}^{p} \varepsilon\left(\varphi^{k-1}\left(\varphi^{n}(x)\right)\right) \leq \sum_{k=0}^{\infty} \varepsilon\left(\varphi^{n+k}(x)\right)
\end{aligned}
$$

for $x \in S$ and $n, p \in \mathbb{N}$.

Now $r_{n}(x):=\sum_{k=0}^{\infty} \varepsilon\left(\varphi^{n+k}(x)\right), n \in \mathbb{N}$, is the remainder of order $n$ of the convergent series (2.3), so $\lim _{n \rightarrow \infty} r_{n}(x)=0$ for all $x \in S$. We conclude that $\left(\varepsilon_{n}(x)\right)_{n \geq 1}$ is a Cauchy sequence, therefore it is convergent since $G$ is a complete metric group. Define the function $f_{0}$ by

$$
f_{0}(x)=\lim _{n \rightarrow \infty} \varepsilon_{n}(x), \quad x \in S .
$$

The relation (2.8), for $p=1$, leads to

$$
d\left(\varepsilon_{n+1}(x), \varepsilon_{n}(x)\right) \leq \sum_{k=0}^{\infty} \varepsilon\left(\varphi^{n+k}(x)\right), \quad n \in \mathbb{N}, x \in S
$$

Taking account of $\varepsilon_{n+1}(x)=g(x)^{-1} \cdot \varepsilon_{n}(\varphi(x))$ and letting $n \rightarrow \infty$ in (2.9) it follows that

$$
d\left(g(x)^{-1} \cdot f_{0}(\varphi(x)), f_{0}(x)\right)=0
$$

which is equivalent to $f_{0}(\varphi(x))=g(x) \cdot f_{0}(x), x \in S$, i.e., $f_{0}$ is a solution of the equation (2.2).

On the other hand, the relations (2.1) and (2.6) lead to

$$
d\left(\varepsilon_{n}(x), f(x)\right) \leq \sum_{k=1}^{n} \varepsilon\left(\varphi^{k-1}(x)\right)
$$


for all $x \in S$ and $n \in \mathbb{N}$, therefore letting $n \rightarrow \infty$ in (2.10) we get

$$
d\left(f_{0}(x), f(x)\right) \leq \Phi(x),
$$

which completes the proof of the existence.

Uniqueness. Assume that for a function $f$ satisfying (2.4) there exist two solutions $f_{1}, f_{2}$ of the equation (2.2) satisfying

$$
d\left(f(x), f_{i}(x)\right) \leq \Phi(x), \quad \forall x \in S, i \in\{1,2\}
$$

and $f_{1} \neq f_{2}$.

Taking into account that $f_{1}, f_{2}$ satisfy (2.2), it follows easily that

$$
f_{i}\left(\varphi^{n}(x)\right)=\prod_{k=1}^{n} g\left(\varphi^{k-1}(x)\right) \cdot f_{i}(x), \quad n \in \mathbb{N}, x \in S, i \in\{1,2\},
$$

and hence

$$
\begin{aligned}
& d\left(f_{1}(x), f_{2}(x)\right) \\
& =d\left(\left(\prod_{k=1}^{n} g\left(\varphi^{k-1}(x)\right)\right)^{-1} \cdot f_{1}\left(\varphi^{n}(x)\right),\left(\prod_{k=1}^{n} g\left(\varphi^{k-1}(x)\right)\right)^{-1} \cdot f_{2}\left(\varphi^{n}(x)\right)\right) \\
& =d\left(f_{1}\left(\varphi^{n}(x)\right), f_{2}\left(\varphi^{n}(x)\right)\right) \\
& \quad \leq d\left(f_{1}\left(\varphi^{n}(x)\right), f\left(\varphi^{n}(x)\right)\right)+d\left(f\left(\varphi^{n}(x)\right), f_{2}\left(\varphi^{n}(x)\right)\right) \\
& \leq 2 \Phi\left(\varphi^{n}(x)\right), \quad x \in S, n \in \mathbb{N} .
\end{aligned}
$$

Since $\lim _{n \rightarrow \infty} \Phi\left(\varphi^{n}(x)\right)=\lim _{n \rightarrow \infty} r_{n}(x)=0, x \in S$, it follows that $f_{1}(x)=f_{2}(x)$, which completes the proof.

The Digamma function $\psi_{0}: \mathbb{R}_{+}^{*} \rightarrow \mathbb{R}$ is defined by (1.6). The Digamma function is frequently called the psi function and it satisfies the Digamma functional equation (1.5) for all $x \in \mathbb{R}_{+}^{*}$. Indeed, we know that $\psi_{0}$ is the unique solution of the functional equation (1.5) which is monotone on $\mathbb{R}_{+}^{*}$ and satisfies $\psi_{0}(1)=-\gamma$, where $\gamma=0.577215 \ldots$ is the Euler-Mascheroni constant (see [1, §6.3] and [21, §6.11.5]).

The gamma function defined by (1.7) satisfies the functional equation

$$
\Gamma(x+1)=x \Gamma(x), \quad \forall x \in \mathbb{R}_{+}^{*} .
$$


If we take the logarithmic values from both sides of the last equation, then we have

$$
\ln \Gamma(x+1)=\ln \Gamma(x)+\ln x, \quad \forall x \in \mathbb{R}_{+}^{*} .
$$

We differentiate each side of the above equality with respect to $x$ to get

$$
\frac{d}{d x} \ln \Gamma(x+1)=\frac{d}{d x} \ln \Gamma(x)+\frac{1}{x}, \quad \forall x \in \mathbb{R}_{+}^{*} .
$$

In view of (1.6), we know that the Digamma function $\psi_{0}$ is a solution of the Digamma functional equation (1.5).

The generalized Hyers-Ulam stability of the Digamma functional equation (1.5) follows from Theorem 2.2 .

Corollary 2.3 Let $\varepsilon: \mathbb{R}_{+}^{*} \rightarrow \mathbb{R}_{+}$be a function with the property

$$
\sum_{n=0}^{\infty} \varepsilon(x+n)=\Phi(x), \quad \forall x \in \mathbb{R}_{+}^{*} .
$$

Then for every function $f: \mathbb{R}_{+}^{*} \rightarrow \mathbb{R}$ satisfying

$$
\left|f(x+1)-f(x)-\frac{1}{x}\right| \leq \varepsilon(x), \quad x \in \mathbb{R}_{+}^{*}
$$

there exists a unique solution $f_{0}: \mathbb{R}_{+}^{*} \rightarrow \mathbb{R}$ of the equation (1.5) such that

$$
\left|f(x)-f_{0}(x)\right| \leq \Phi(x), \quad \forall x \in \mathbb{R}_{+}^{*}
$$

Proof. Take $S=\mathbb{R}_{+}^{*}, \varphi(x)=x+1, G=\mathbb{R}$ with the usual addition and $d$ the Euclidian metric on $\mathbb{R}$ and $g(x)=1 / x, x \in \mathbb{R}_{+}^{*}$. Then the result follows in view of Theorem 2.2 .

\section{References}

[1] M. Abramowitz, I. A. Stegun, Handbook of Mathematical Functions with Formulas, Graphs, and Mathematical Tables, Dover Publ., New York, 1965 . 
[2] R.P. Agarwal, B. Xu, W. Zhang, Stability of functional equations in single variable, J. Math. Anal. Appl., 288 (2003), 852-869.

[3] J. Brydak, On the stability of the functional equation $\varphi[f(x)]=$ $g(x) \varphi(x)+F(x)$, Proc. Amer. Math. Soc., 26 (1970), 455-460.

[4] J. Brzdek, N. Brillouët-Bellout, K. Ciepliński, On some recent developments in Ulam's type stability, Abstr. Appl. Anal. 2012 (2012), Art. ID 716936 .

[5] J. Brzdek, J. Chudziak, Zs. Páles, A fixed point approach to stability of functional equations, Nonlinear Anal., 74 (2011), 6728-6732.

[6] J. Brzdek, D. Popa, B. Xu, The Hyers-Ulam stability of linear equations of higher orders, Acta Math. Hungar., 120 (2008), 1-8.

[7] J. Brzdek, S.-M. Jung, A note on stability of an operator equation of the second order, Abstr. Appl. Anal., 2011 (2011), Art. ID 602713, 15 pp.

[8] J. Brzdek, D. Popa, B. Xu, On approximate solutions of the linear functional equation of higher order, J. Math. Anal. Appl., 373 (2011), 680-689.

[9] J. Brzdek, D. Popa, B. Xu, Note on nonstability of the linear functional equation of higher order, Comput. Math. Appl., 62 (2011), 2648-2657.

[10] J. Brzdek, D. Popa, B. Xu, Selections of set-valued maps satisfying a linear inclusion in a single variable, Nonlinear Anal., 74 (2011), 324-330.

[11] E. Castillo and M. R. Ruiz-Cobo, Functional Equations and Modelling in Science and Engineering, Marcel Dekker, New York/Basel/Hong Kong, 1992.

[12] L. Cesaro, Optimization Theory and Applications. Problems with Ordinary Differential Equations, Springer, New York/Heidelberg/Berlin, 1983.

[13] S. Czerwik, Functional Equations and Inequalities in Several Variables, World Scientific, River Edge, NJ, 2002.

[14] S.-M. Jung, On the modified Hyers-Ulam-Rassias stability of the functional equation for gamma function, Mathematica (Cluj), 39(62) (1997), 235-239. 
[15] S.-M. Jung, Hyers-Ulam-Rassias Stability of Functional Equations in Nonlinear Analysis, Springer Optimization and its Applications, Vol. 48, Springer, New York, 2011.

[16] M. Kuczma, Functional Equations in a Single Variable, Państwowe Wydawnictwo Naukowe, Warszawa, 1968.

[17] P. M. Pardalos and T. F. Coleman (eds.), Lectures on Global Optimization, Fields Institute Communications, Amer. Math. Soc., 2009.

[18] G. Polya, G. Szegö, Aufgaben und Lehrsätze aus der Analysis I, Julius Springer, Berlin, Germany, 1925.

[19] T. T. Rockafellar, Convex Analysis, Princeton Univ. Press, Princeton, 1972.

[20] T. Trif, On the stability of a general gamma-type functional equation, Publ. Math. Debrecen, 60 (2002), 47-61.

[21] D. Zwillinger, Standard Mathematical Tables and Formulae (31th Ed.), Chapman \& Hall/CRC, 2003. 